\title{
Biallelic variants in YRDC cause a developmental disorder with progeroid features
}

\author{
Julia Schmidt ${ }^{1}$ (1) Jonas Goergens ${ }^{2,10} \cdot$ Tatiana Pochechueva $^{3} \cdot$ Annika Kotter $^{4}$ - Niko Schwenzer ${ }^{3,15} \cdot$ Maren Sitte $^{5}$. \\ Gesa Werner ${ }^{1}$. Janine Altmüller ${ }^{6,16,17}$ • Holger Thiele ${ }^{6} \cdot$ Peter Nürnberg $^{6}$. Jörg Isensee ${ }^{9}$ Yun Li ${ }^{1}$. Christian Müller ${ }^{1}$. \\ Barbara Leube $^{7}$. H. Christian Reinhardt ${ }^{8} \cdot$ Tim Hucho $^{9} \cdot$ Gabriela Salinas $^{5} \cdot$ Mark Helm $^{4}$ • Ron D. Jachimowicz ${ }^{2,10,11,12}$. \\ Dagmar Wieczorek ${ }^{7} \cdot$ Tobias Kohl $^{3,13} \cdot$ Stephan E. Lehnart ${ }^{3,13,14,15,18,19} \cdot$ Gökhan Yigit $^{1} \cdot$ Bernd Wollnik $^{1,15}$
}

Received: 6 May 2021 / Accepted: 13 August 2021 / Published online: 20 September 2021

(c) The Author(s) 2021

\begin{abstract}
The highly conserved YrdC domain-containing protein (YRDC) interacts with the well-described KEOPS complex, regulating specific tRNA modifications to ensure accurate protein synthesis. Previous studies have linked the KEOPS complex to a role in promoting telomere maintenance and controlling genome integrity. Here, we report on a newborn with a severe neonatal progeroid phenotype including generalized loss of subcutaneous fat, microcephaly, growth retardation, wrinkled skin, renal failure, and premature death at the age of 12 days. By trio whole-exome sequencing, we identified a novel homozygous missense mutation, c.662T >C, in YRDC affecting an evolutionary highly conserved amino acid (p.Ile221Thr). Functional characterization of patient-derived dermal fibroblasts revealed that this mutation impairs YRDC function and consequently results in reduced $\mathrm{t}^{6} \mathrm{~A}$ modifications of tRNAs. Furthermore, we established and performed a novel and highly sensitive 3-D Q-FISH analysis based on single-telomere detection to investigate the impact of YRDC on telomere maintenance. This analysis revealed significant telomere shortening in YRDC-mutant cells. Moreover, single-cell RNA sequencing analysis of YRDC-mutant fibroblasts revealed significant transcriptome-wide changes in gene expression, specifically enriched for genes associated with processes involved in DNA repair. We next examined the DNA damage response of patient's dermal fibroblasts and detected an increased susceptibility to genotoxic agents and a global DNA double-strand break repair defect. Thus, our data suggest that YRDC may affect the maintenance of genomic stability. Together, our findings indicate that biallelic variants in $Y R D C$ result in a developmental disorder with progeroid features and might be linked to increased genomic instability and telomere shortening.
\end{abstract}

\section{Introduction}

Segmental progeroid syndromes are rare congenital disorders characterized by signs and symptoms of premature or accelerated aging. Typical features of progeroid syndromes include, e.g., lipodystrophy, growth retardation, hair loss, brittle bones, atherosclerosis, and hearing loss. Several of these progeroid disorders show overlapping phenotypes, which makes specific clinical diagnosis often challenging. Most progeroid syndromes known so far belong to a very heterogeneous group of disorders caused by autosomal

Julia Schmidt

julia.schmidt1@med.uni-goettingen.de

$\triangle$ Bernd Wollnik

bernd.wollnik@med.uni-goettingen.de

Extended author information available on the last page of the article dominantly or recessively inherited mutations in a single gene. Yet identified cellular pathways and molecular pathomechanisms underlying premature aging mainly affect DNA damage repair processes, nuclear membrane dynamics, chromatin structure or transcription and, thereby, have an impact on various aspects of cell viability (Gordon et al. 2014; Carrero et al. 2016). Genomic instability as well as telomere shortening have been identified as particularly relevant for aging-associated processes (Harley et al. 1990; Blackburn 1991; Lange 2005; Martínez and Blasco 2011). In many aspects, the molecular characteristics of progeroid syndromes seem to be similar to those of "physiological" aging. Therefore, studying conditions of premature aging will help to reveal unknown underlying causal mechanisms and to develop potentially new treatments for more frequent age-associated diseases (Lessel and Kubisch 2019). 
Previous studies suggest that YRDC (YrdC domaincontaining protein; OMIM\#612276), the human homolog of yeast Sua5, contributes to various central cellular functions. First, YRDC has been described to be necessary for adding specific tRNA modifications that are essential to ensure the accuracy of protein synthesis. YRDC in combination with the Kinase, Endopeptidase and Other Proteins of small Size (KEOPS) protein complex synthesizes the universally conserved threonylcarbamoylation of the N6 nitrogen of the adenosine at the tRNA position $37\left(\mathrm{t}^{6} \mathrm{~A}\right)$ (Lin et al. 2018). Mutations in genes encoding subunits of the KEOPS complex lead to decreased $t^{6} \mathrm{~A}$ levels and cause Galloway-Mowat syndrome (GAMOS, OMIM\#251300), a rare autosomal recessive condition characterized by the association of early onset nephrotic syndrome and microcephaly with central nervous system anomalies (Braun et al. 2017). Second, the KEOPS complex and Sua5 have been identified to promote telomere maintenance, an essential process for safeguarding genomic stability (Downey et al. 2006; Meng et al. 2010). Third, He et al. (2019) identified that the KEOPS complex affects both DNA damage response (DDR) and repair, especially homologous recombinationmediated DNA repair, independently of its $\mathrm{t}^{6} \mathrm{~A}$ synthesis function. Very recently, two missense mutations in $Y R D C$ were associated with GAMOS phenotypes, suggesting overlapping functional roles of YRDC and the KEOPS complex in cellular processes (Arrondel et al. 2019).

Here, we report on a patient with a severe developmental disorder with progeroid features in whom we identified a novel causative, homozygous $Y R D C$ mutation. Analysis of fibroblasts revealed reduced levels of tRNA modification, telomere shortening, and impaired DDR.

\section{Results}

\section{Clinical report}

The index patient II.5 was born after $37+0$ weeks of gestation to healthy, distantly consanguineous parents from northern Iraq origin (Fig. 1). His birth weight was $1740 \mathrm{~g}$ $(-3.1 \mathrm{SD})$, his birth length $46 \mathrm{~cm}(-1.8 \mathrm{SD})$, and his head circumference (OFC) at birth $29 \mathrm{~cm}(-3.6 \mathrm{SD})$. After birth, he developed tonic-clonic seizures and primary liver and kidney dysfunction. Newborn screening revealed hypothyroidism (TSH: $996.10 \mu \mathrm{U} / \mathrm{ml}$; fT3: $0.13 \mathrm{ng} / \mathrm{dl}$ ) and he received intravenous $\mathrm{L}$-thyroxine. He required assisted ventilation. Furthermore, he showed generalized loss of subcutaneous fat with a striking progeroid appearance, hypertrichosis, arachnodactyly and adducted thumbs as well as facial dysmorphisms including low-set and large appearing ears, micro- and retrognathia, long, smooth philtrum, and wrinkled skin. He had congenital intestinal malrotation and needed surgery due to meconium ileus. After surgery, he developed renal failure and intracranial hemorrhage and died at the age of 12 days. Family history revealed two healthy older sisters, II.2 and II.3, and a brother who died few hours after birth (II.1). In addition, the mother had one early miscarriage (II.4).

\section{Identification of a homozygous YRDC mutation}

Initial genetic testing for progeroid syndromes using a custom-designed multigene panel including 87 known diseaseassociated genes was unremarkable. Therefore, trio wholeexome sequencing (WES) was performed on DNA extracted from blood of the patient II.5 and his unaffected parents, I.1 and I.2, using the Agilent SureSelectXT Human All Exon V7 enrichment kit on an Illumina HiSeq4000 sequencer. We analyzed and filtered the trio WES data using the exome and genome analysis pipeline "Varbank 2.0" (https://varba nk.ccg.uni-koeln.de/varbank2) of the Cologne Center for Genomics (CCG, University of Cologne, Germany), and we obtained a mean coverage of $60-65$ and $96.1-96.3 \%$ of target were covered more than $10 \times$. Trio-based WES is an effective diagnostic tool for identification of causative variants in individuals with developmental disorders (Palomares-Bralo et al. 2017). de novo variants are known to be one of the most common genetic causes of developmental disorders (AcunaHidalgo et al. 2016). Both parents of the index patient II.5 were unaffected, so our analysis included different modes of inheritance. We identified only a single heterozygous de novo variant, which was excluded as causative variant based on population frequency and lacking evidence for functional impairment on the affected protein (Supplementary Table S1). Based on the family history and the reported parental consanguinity, we next focused on homozygous variants consistent with a recessive transmission. We identified only one single homozygous variant not present in any current database of human genetic variations including the gnomAD (https://gnomad.broadinstitute.org) database (last access date 12/01/2021, (Karczewski et al. 2019)) and predicted to have a severe impact on protein structure. All de novo and rare biallelic or hemizygous variants are listed in the supplement (Supplemental Table S1). The homozygous missense variant, c.662T $>\mathrm{C}$, was located in exon 4 of the YRDC gene (Fig. 1D) and leads to the substitution of an evolutionary highly conserved isoleucine at the amino acid position 221 by threonine (p.Ile221Thr). The variant was predicted as disease-causing by MutationTaster (http://www. mutationtaster.org), damaging by SIFT (https://sift.bii.a-star. edu.sg), probably damaging by PolyPhen-2 (http://genetics. bwh.harvard.edu/pph2), and has a CADD (https://cadd.gs. washington.edu) score of 27.7, indicating deleteriousness of this variant. The mutation was embedded in the largest autosomal homozygous region $(>5 \mathrm{Mb}$ ) determined from 
A
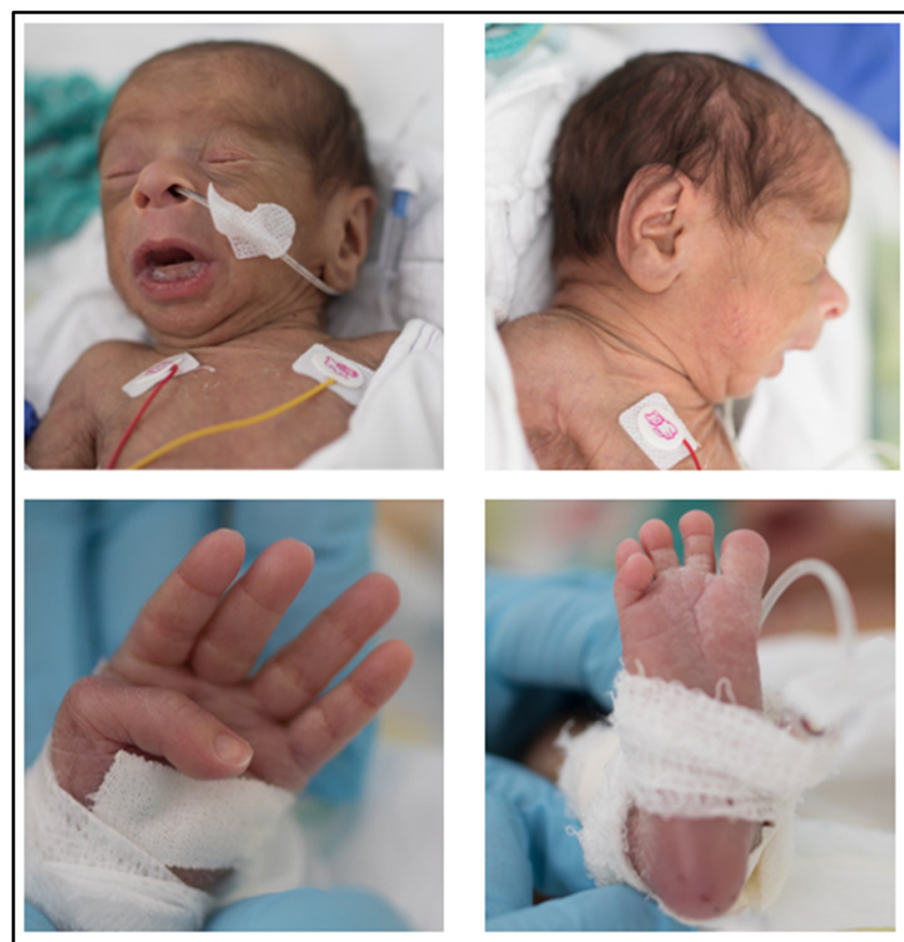

$\mathbf{E}$



$\mathbf{F}$

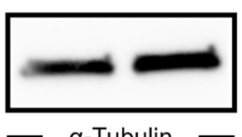

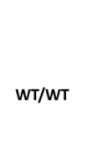

YRDC
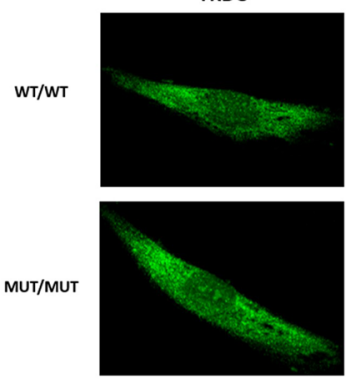

DAPI
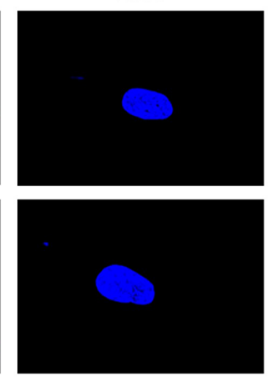

B

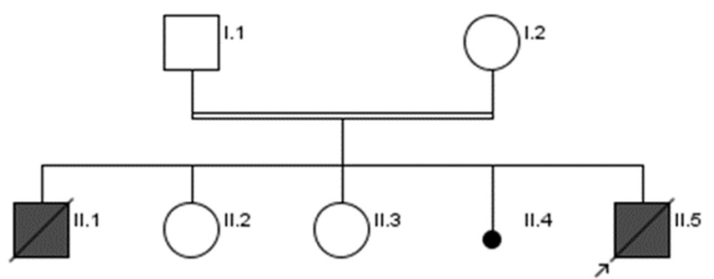

C

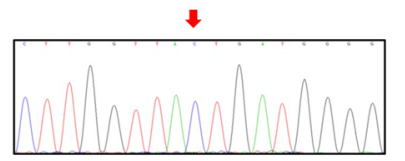

I.1: MUT/WT



I.2: MUTNT

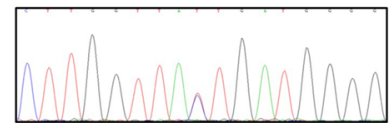

D
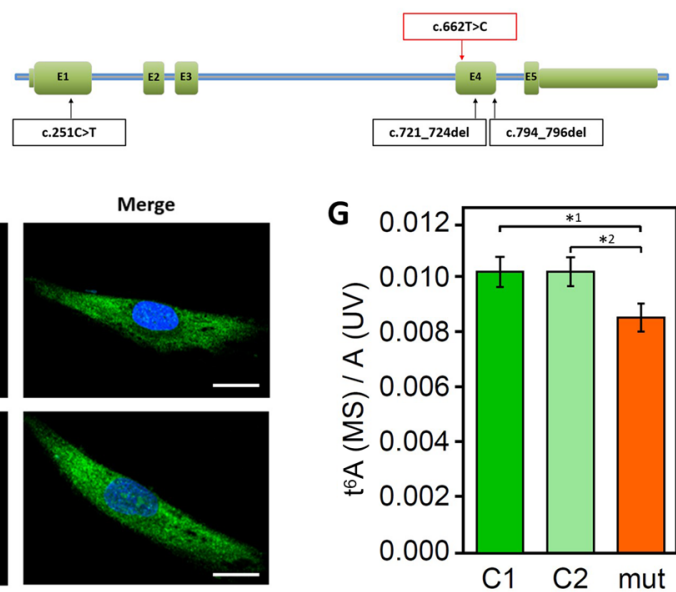

G

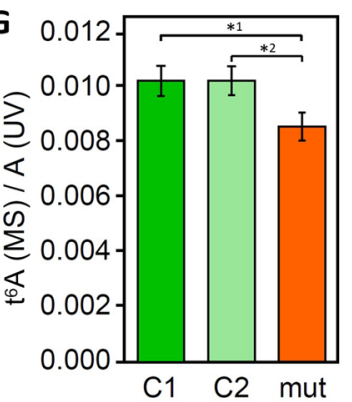

Fig. 1 Identification of a homozygous mutation in $Y R D C$ in a newborn with a progeroid phenotype. A Clinical characteristics of the index patient II.5 at the age of 5 days. Physical features included generalized loss of subcutaneous fat with a progeroid appearance, hypertrichosis, low-set and large appearing ears, micro- and retrognathia, long, smooth philtrum, wrinkled skin, arachnodactyly and adducted thumbs. B Family pedigree of the consanguineous family from northern Iraq. Index patient is indicated by arrow. C Electropherograms of the identified $Y R D C$ mutation (red arrow) confirming homozygous state in the affected sibling II.5 and heterozygous carrier status of both parents, I.1 and I.2 (MUT mutation, WT wild type). D Schematic view of $Y R D C$ and localization of the identified mutations. Red arrow indicates the $Y R D C$ mutation identified within this study, previously identified mutations are indicated by black arrows. E Western blot analysis was performed on whole-cell lysates from healthy control fibroblasts (WT/WT) and patient (II.5)-derived fibroblasts (MUT/ MUT) using anti-YRDC antibody (upper panel). Anti- $\alpha$-Tubulin antibody was used as a loading control (lower panel). F Analysis of subcellular localization of WT (upper panel) and mutant (lower panel) YRDC by immunofluorescence staining in patient-derived (MUT) and control (WT) fibroblasts using anti-YRDC-antibodies (green). Nuclei were counterstained with DAPI (blue). Scale bar, $20 \mu \mathrm{m}$. G LC-MS/MS-based quantification of $\mathrm{t}^{6} \mathrm{~A}$ levels in patient-derived fibroblasts (MUT) and two different controls ( $\mathrm{C} 1$ and $\mathrm{C} 2)$. Technical triplicates have been performed on one biological sample each. Levels are shown as MS signal of $\mathrm{t}^{6} \mathrm{~A}$ divided by UV signal of adenosine. $p$ values were calculated using two-sided $t$ test: $* 1 p=0.0285$, $*^{2} p=0.0259$ the WES data set and was found in heterozygous state in both parents. Sanger sequencing was used to validate the WES data and to prove that the healthy siblings, II.2 and II.3, do not carry the variant in the homozygous state. DNA from the deceased brother was not available for genetic testing (Fig. 1B).

\section{Effects of the YRDC mutation on protein stability, localization and tRNA modification}

To examine the cellular consequences of the p.Ile221 Thr mutation on protein stability and expression levels, we compared the YRDC protein levels in primary dermal fibroblasts 
from patient II.5 and fibroblasts from a matched healthy control. Western blot analysis showed comparable expression levels of YRDC in both control and patient fibroblasts (Fig. 1E). Next, we analyzed the subcellular localization of wild type (WT) and mutant (MUT) YRDC by immunostaining. YRDC showed a widely diffused intracellular localization in both, WT and MUT cells (Fig. 1F). Taken together, these data suggest that the missense variant p.Ile221 Thr in YRDC does not affect its stability or subcellular localization in patient fibroblasts.

YRDC has been described to be essential for the synthesis of $\mathrm{t}^{6} \mathrm{~A}$ at position $\mathrm{A} 37$ of several tRNAs. To assess the effect of the mutation on the level of $t^{6} \mathrm{~A}$ in tRNAs, total RNA was extracted from patient's skin fibroblasts and subjected to LC-MS/MS analysis. As expected, the global $t^{6} \mathrm{~A}$ level of the patient showed a significant decrease of $16.3 \%$ (Fig. 1G) compared to WT samples. This shows that the homozygous c.662T > C, p.Ile221Thr mutation in YRDC affects the $\mathrm{t}^{6} \mathrm{~A}$ biosynthesis providing additional functional evidence for the causative nature of this homozygous missense mutation.

\section{Q-FISH-based evaluation of telomere length}

It is well established that telomere shortening and agingrelated processes are strongly associated with each other. However, the detailed mechanisms of, e.g., accelerated telomere shortening in monogenic progeria phenotypes are largely unknown. Telomere brightness by FISH staining has been validated as a solid measure of telomere lengths (Ourliac-Garnier and Londoño-Vallejo 2017) with a linear brightness-to-telomere relationship (Martens et al. 1998; Poon et al. 1999). We, therefore, established and performed a novel and highly sensitive in situ Q-FISH analysis in patient fibroblasts (MUT) relying on a 2-step procedure. First superresolution STimulated Emission Depletion (STED) microscopy was applied to in situ FISH samples (Fig. 2A) and used to confirm sufficient spatial separation of in situ FISH-labeled telomeres by at least $200 \mathrm{~nm}$. As a second step, confocal imaging produced z-stacks of whole nuclei, and consequently individual telomeres were identified and analyzed automatically with image processing algorithms (Fig. 2B). Importantly, as a prerequisite for the comparative analysis, we have confirmed equal likelihoods of telomere detection in all samples regardless of average telomere brightness (Fig. 2C). Our analysis of telomere brightness revealed a significant decrease $(p<0.01)$ in telomere brightness in MUT as compared to an age-matched control (WT1) and a healthy adult (WT2) (Fig. 2D) with both matching numbers of passages. This corresponds to on average approximately $26 \%$ telomere shortening of chromosomes in patient cells, with shortening being seemingly proportional to regular individual telomere lengths. Notably, we observed a significant increase in the occurrence of short (detected as dark) telomeres (Fig. 2E). To further analyze whether this telomere shortening is associated with increased cellular senescence, we measured the senescenceassociated beta-galactosidase activity in patient and control fibroblasts. We observed an increased number of beta-galactosidase-positive patient cells corresponding to a higher proportion of senescent cells ( $23.7 \%$ in patient cells compared to $14.5 \%$ in control fibroblasts), which in both cases could be elevated by approximately $15 \%$ by treatment with $\mathrm{H}_{2} \mathrm{O}_{2}$ (39.6\% in patient cells compared to $28.7 \%$ in control fibroblasts) (Supplementary Figure S2).

\section{Specific gene expression signatures based on single-cell RNA sequencing (scRNA-seq)}

ScRNA-seq can be a powerful tool for analyzing changes in gene expression at the cellular level and elucidating novel biological mechanisms. To explore the effect of the homozygous p.Ile221Thr mutation in YRDC on the transcriptome, we performed single-cell RNA sequencing on patient fibroblasts and two sex- and age-matched controls. We used Gene Set Enrichment Analysis (GSEA) to determine whether particular subsets of genes were differentially expressed. Of specific interest was the finding that among the top 10 enriched pathways in YRDC mutant cells we found "Global Genome Nucleotide Excision Repair", "Homology Directed Repair" and "HDR through Homologous Recombination (HRR) or Single Strand Annealing (SSA)" (Fig. 3) to be altered. Even though the normalized enrichment scores were low, the prevalence of enriched pathways linked to DNA repair mechanisms suggests a possible interaction between YRDC function and maintenance of genomic stability. Genome instability is a cellular hallmark observed in cells of patients with different forms of premature aging syndromes, such as Cockayne syndrome, Ataxia-telangiectasia and Bloom syndrome (Wu and Hickson 2003; Trapp et al. 2007; Shiloh and Lederman 2017). Interestingly, YRDC has very recently been identified in CRISPR/Cas9 screens as important for DNA damage response in human cells (Olivieri et al. 2020).

\section{Altered DNA damage response (DDR) and DNA double-strand break (DSB) repair}

To gain further insight into whether different pathways involved in DDR and DNA repair are affected by the identified mutation in $Y R D C$, we next examined the response of patient's dermal fibroblasts to different genotoxic agents. MUT and WT fibroblasts were treated with etoposide, cisplatin, camptothecin (CPT) or hydroxyurea (HU) and cell viability was determined for different timepoints after treatment (Fig. 4A-D). Interestingly, MUT fibroblasts showed an increased susceptibility to all employed genotoxic agents compared to WT controls, with the strongest effect observed 

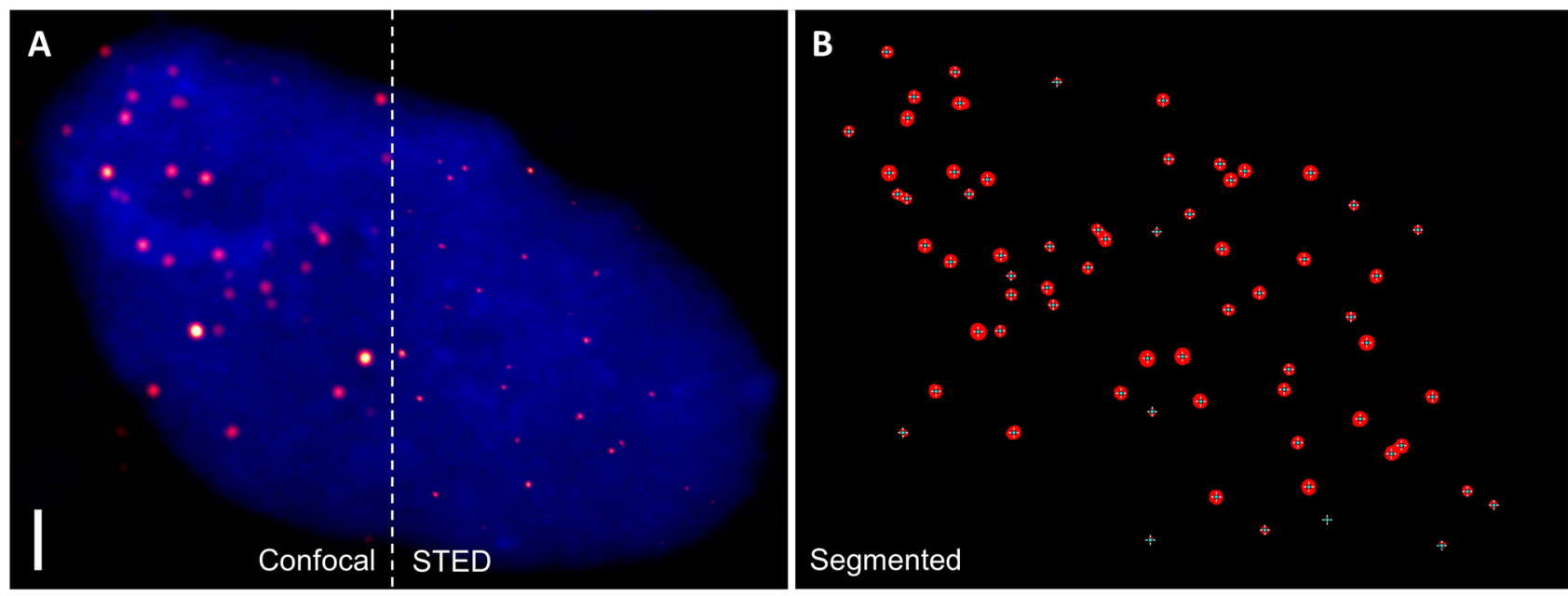

\section{Telomeres per cell}

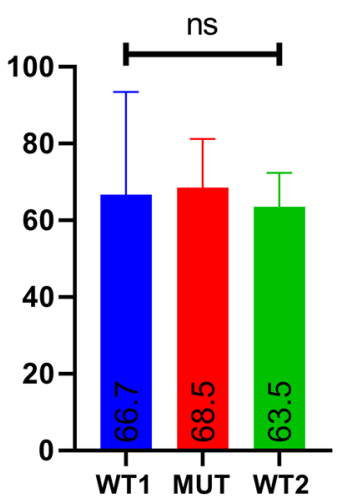

Telomere brightness

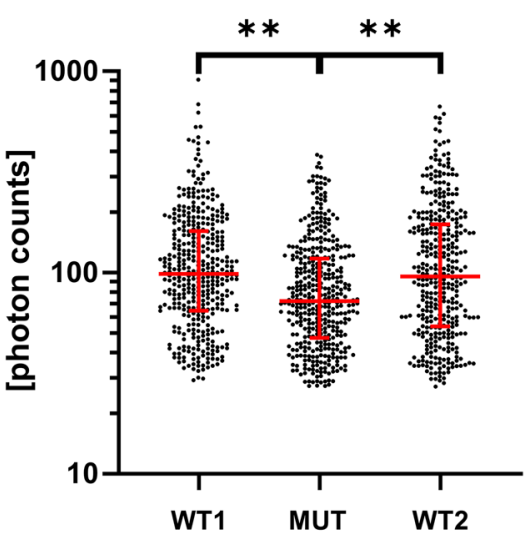

$\mathbf{E}$

Lognormal distributions of telomere brightness

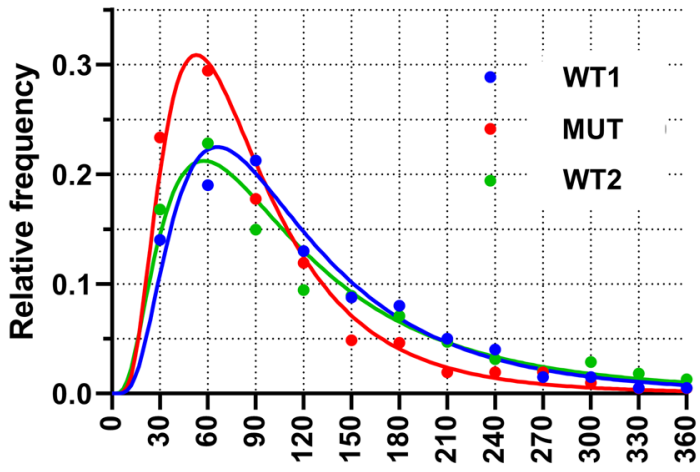

Telomere brightness
Fig. 2 Q-FISH analysis of telomeres in patient fibroblasts and two controls. A STED imaging of a TelC-Star-635P FISH-staining (Redhot LUT) with DAPI (blue, confocal) counterstain. Z-projection of STED imaging confirms that telomeres do not cluster and are distant enough to be resolved by confocal z-stacks. Scale bar $=2 \mu \mathrm{m}$. B Thresholder confocal image showing detected telomeres with marked center coordinates. Even partially overlapping spots could be resolved across a wide range of signal amplitudes. C Detection efficiency for telomeres is similar in all experimental groups despite telomere shortening, as shown by similar numbers of detected telomeres per cell after detection and fit process $(n=66.7 \pm 26.8,68.5 \pm 12.7,63.5 \pm 8.9$, mean \pm std). D Telomere brightness is significantly reduced in patient fibroblasts (MUT). Each scatter includes the data of 6 nuclei. The nuclei of each respective sample had a mean $( \pm$ std) telomere brightness of $126.3 \pm 13.2$ (WT1), 93.3 \pm 14.4 (MUT), and 132.9 \pm 22.0 (WT2) photon counts. Red lines indicate median and IQR. Stars indicate significance $(p<0.01)$ by nested one-way ANOVA of log-transformed data, sub grouped by nucleus. E Dark telomeres appear more frequently in MUT fibroblasts with altered genetic background. Telomere brightness data follows a lognormal probability function after treatment with cisplatin or CPT (Fig. 4B, C), possibly suggesting impaired DNA DSB repair in MUT cells (Dasari and Tchounwou 2014).

To further characterize involved DNA repair pathways in YRDC mutant cells, we employed automated immunofluorescence microscopy to quantify nuclear $\gamma \mathrm{H} 2 \mathrm{AX}$ foci, as a surrogate for unrepaired DNA lesions, as well as 53BP1 and RAD51 foci, which are markers for non-homologous end joining (NHEJ) and homologous recombination (HRR) pathways of DSB repair, respectively (Jachimowicz et al. 2019). Without treatment, we did not detect any significant differences in the numbers of $\gamma \mathrm{H} 2 \mathrm{AX}, 53 \mathrm{BP} 1$, and RAD51 foci in MUT compared to WT cells. In response to etoposide, we observed a significantly delayed clearance of $\gamma \mathrm{H} 2 \mathrm{AX}$ foci in MUT compared to WT cells (Fig. 4E, left panel). In contrast to the etoposide response, MUT cells treated with cisplatin, $\mathrm{CPT}$ or HU showed a continuous increase in $\gamma \mathrm{H} 2 \mathrm{AX}$ foci throughout the 24-h observation period compared to WT cells (Fig. 4F-H, left panel). Furthermore, MUT cells generally displayed higher numbers of 53BP1 foci following treatment with all four genotoxic agents compared to WT cells (Fig. 4E-H, middle panel). This difference was especially pronounced $24 \mathrm{~h}$ after treatment coinciding with high levels of $\gamma \mathrm{H} 2 \mathrm{AX}$ foci in MUT cells. With the exception of 
Fig. 3 Whole transcriptome analysis based on single-cell RNA sequencing reveals different gene expression in YRDC-mutant fibroblasts. Gene Ontology (GO) and Reactome enrichment analysis showing the top 10 enriched pathways in patient fibroblasts compared to two sex- and age-matched controls. Categories related to DNA repair are highlighted in dark grey

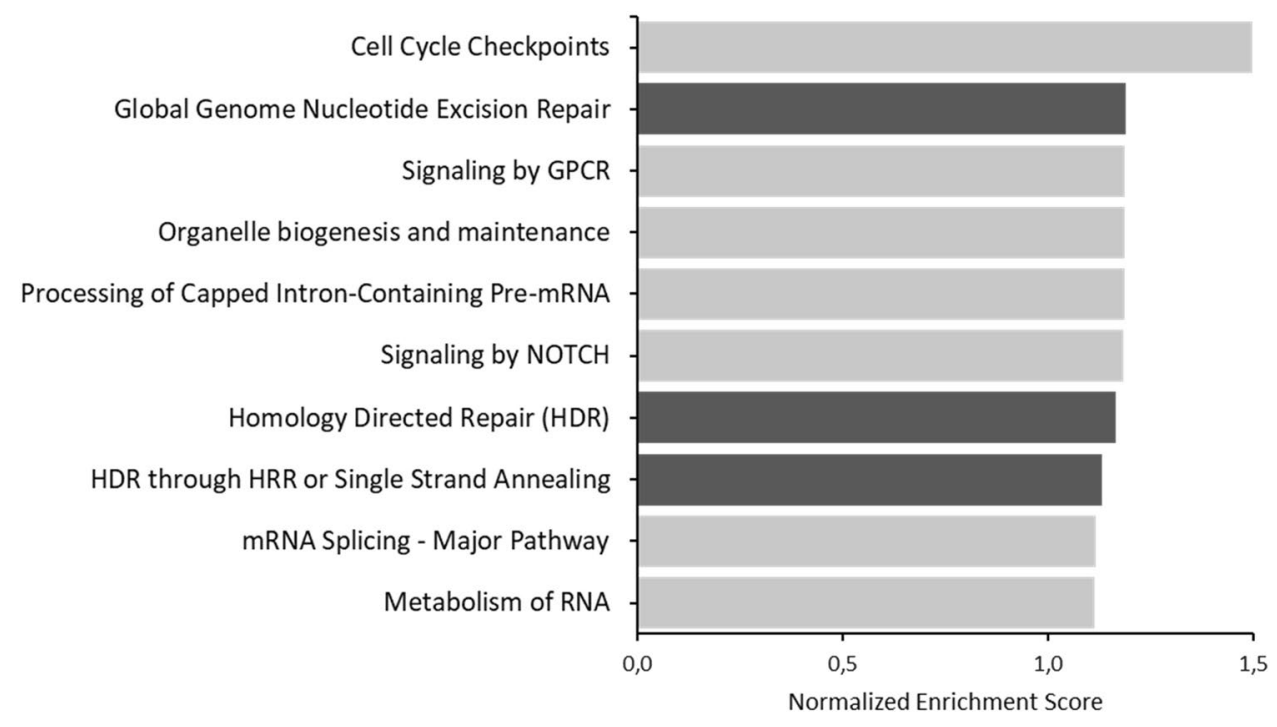

etoposide-treated cells, MUT cells also displayed increased numbers of RAD51 foci following DNA damage induction (Fig. 4E-H, right panel). Corresponding immunofluorescence microscopy images are included in the supplement (Supplemental Figures S3-S5). Taken together, these results combined with the ScRNA-seq data may suggest that the homozygous p.Ile221Thr mutation in YRDC confers hypersensitivity to DNA-damaging agents and leads to a global DNA DSB repair defect, potentially affecting both HRR and NHEJ.

\section{Discussion}

Here, we present a patient with a severe developmental disorder with progeroid features caused by a novel homozygous missense mutation in YRDC. Our functional studies indicate that mutant YRDC results in defective tRNA modification, telomere shortening and increased genomic instability, thereby, giving first insights into the molecular pathogenesis of this novel accelerated aging phenotype.

YRDC is a highly conserved protein interacting with the well-described KEOPS complex (Lin et al. 2018). Mutations in genes encoding subunits of the KEOPS complex cause Galloway-Mowat syndrome (GAMOS, OMIM\#251300), a rare autosomal recessive condition characterized by the association of early onset nephrotic syndrome and microcephaly with central nervous system anomalies (Braun et al. 2017). Although patients with mutations in KEOPS share some phenotypic features with the individual reported in our study, such as, e.g., renal abnormalities or seizures, progeroid features as presented by our patient were not observed in GAMOS patients with mutations in KEOPS complex components. A recently published study reported a family with an affected child carrying compound heterozygous $Y R D C$ mutations (missense mutation (c.251C > T, p.Ala84Val) and a 4-base pair deletion leading to a frameshift (c.721_724del, p.Val241Ilefs*72)) and a further family with two affected children carrying a homozygous in-frame deletion of leucine 265 (c.794_796del, p.Leu265del) (Arrondel et al. 2019). Similar to our patient, all these individuals died early (oldest age of death 15 months) and they shared some additional specific clinical features, such as congenital hypothyroidism, renal failure, and facial dysmorphism. Like our patient, the two affected siblings reported by Arrondel et al. showed arachnodactyly and primary microcephaly, while these features were not present in the single patient of the cited study. Arrondel et al. (2019) suggested that mutations in YRDC cause an "extremely severe form of GAMOS". The main clinical features are summarized in Table 1. Considering the phenotypic overlap of all four patients, we postulate that patients with homozygous or compound heterozygous $Y R D C$ mutations should be viewed as a distinct entity based on the specific phenotypic features of congenital hypothyroidism, progeroid appearance and premature death.

Our analysis of the functional consequences of the identified p.Ile221Thr mutation in YRDC indicated that mutant YRDC might result in decreased threonylcarbamoylation of the N6 nitrogen of the adenosine at the tRNA position 37 $\left(t^{6} \mathrm{~A}\right)$. Previous studies have shown that YRDC acts in this process together with the KEOPS complex, and mutations in KEOPS proteins as well as in YRDC have been linked to decreased $\mathrm{t}^{6} \mathrm{~A}$ levels (El Yacoubi et al. 2009; Srinivasan et al. 2011; Perrochia et al. 2013; Lin et al. 2018). The reduction of $\mathrm{t}^{6} \mathrm{~A}$ modifications that we observed in patientderived fibroblasts strongly supports causality of the identified missense variant p.Ile221Thr in YRDC. Still, based on the different phenotypic presentation of GAMOS patients with mutations in KEOPS proteins and patients with YRDC mutations, it is likely that YRDC is involved in additional 
cellular processes which are not depending on the KEOPS complex.

As we observed progeroid features in our patient, we focused on the putative relevance of YRDC in aging processes, reflected by its role in telomere maintenance and DDR. Arrondel et al. (2019) reported that the telomere length in their patients with YRDC mutations was not affected. Their results were based on a telomere restriction fragment assay and traditional Southern blot analysis, which revealed a higher telomere length in affected children compared to their parents. A solid method to measure the telomere length is Q-FISH analysis (Martens et al. 1998; Poon et al. 1999; Ourliac-Garnier and Londoño-Vallejo 2017). Here, we performed a novel and highly sensitive Q-FISH analysis to investigate the impact of mutant YRDC on telomere maintenance. Performing three-dimensional (3D) confocal imaging with $200 \mathrm{~nm}$ axial step size followed by 3D image analysis we were able to circumvent most of the inherent disadvantages of conventional Q-FISH methods, such as, e.g., insufficient separation of adjacent and superimposed telomeres and frequent out-of-focus detections (Poon et al. 1999). We were able to detect and quantify most individual telomeres in the appropriate respective imaging planes and thus obtained a remarkable cellular telomere coverage of over $70 \%$. This confirms the strength of our 3D imaging and analysis approach, resulting in more reliable and accurate readout of individual telomere brightness. Our analysis revealed a significant decrease in telomere brightness in patient's fibroblasts, indicating significant telomere shortening in the patient cells. This is the first time that microscopy-based Q-FISH analysis of individual telomeres was used to determine telomere shortening in a human progeroid phenotype. Importantly, our data confirms that telomeres were detected at same efficiencies in all groups regardless of the average telomere brightness, confirming the robustness of this approach to identify telomeres individually in three dimensions. In contrast to the study by Arrondel et al., we compared telomere length to an agematched control instead of parental control samples, which might explain the differences in the age-associated outcome of the analyses.

However, the exact mechanistic link between YRDC and telomere length remains to be elucidated in comprehensive future studies and rescue experiments are required to confirm our findings. Some previous studies have speculated that the influence of YRDC/Sua5 and the KEOPS complex on telomere length might be attributed to the lack of $\mathrm{t}^{6} \mathrm{~A}$ modification (Daugeron et al. 2011; El Yacoubi et al. 2011; Perrochia et al. 2013; Huang et al. 2019). In contrast, Downey et al. (2006) hypothesized that the KEOPS complex promotes both telomere uncapping and telomere elongation. Meng et al. $(2009,2010)$ showed that SUA5 also positively regulates telomere maintenance. Liu et al. (2018) supported with their results a model in which Sua5 and the KEOPS complex are essential for telomere homeostasis independent of its t6A biosynthesis activity and suggested that KEOPS regulates telomere length by promoting G-overhang generation. Although its precise action is still elusive, our data provides additional support for the assumption that YRDC is linked to molecular mechanisms underlying accelerated aging processes.

In addition, we analyzed the senescence-associated $\beta$-galactosidase activity and observed that patient-derived fibroblasts show an increased number of $\beta$-galactosidasepositive cells corresponding to a higher proportion of senescent cells. Cellular senescence is defined as a stable state of cell cycle arrest (Rhinn et al. 2019). Besides the well-established role of accumulating senescent cells in aging and age-associated conditions, senescence has been linked to proper embryonic development (Gal et al. 2021), and positive senescence-associated $\beta$-galactosidase staining can be observed at multiple locations in whole-mount mice embryos (Muñoz-Espín et al. 2013). Recent studies confirmed that cellular senescence contributes to normal tissue patterning and organ development during embryogenesis and later in life is essential for tissue repair and regeneration (Gal et al. 2021; Di Micco et al. 2021; Muñoz-Espín und Serrano 2014). Accumulation of senescent cells might directly impair tissue proliferation (Rhinn et al. 2019) and thereby contribute to the severe developmental anomalies observed in our patient.

Furthermore, ScRNA-seq revealed an upregulation of DDR pathways possibly indicating genomic instability in YRDC mutant fibroblasts. Keeping in mind that enrichment scores were low, these results need to be interpreted carefully. For validation, we performed additional functional assays using patient fibroblasts. This further analysis showed a generally increased susceptibility to DNA-damaging agents and a global DNA DSB repair defect, affecting both HRR and NHEJ. Impairment of DDR mechanisms is commonly observed in patients with different progeroid syndromes including, e.g., Hutchinson-Gilford progeria syndrome, Werner syndrome, or Cockayne syndrome. Interestingly, Braun et al. (2017) observed increased activation of DDR pathways resulting in reduced cellular viability after knockdown of KEOPS complex subunits, indicating that YRDC might contribute to DDR in interaction with the KEOPS complex. Our results are consistent with the very recently published CRISPR/Cas9 screen, in which Olivieri et al. (2020) showed as well that YRDC might be an important factor for DNA damage response and thereby promoting DNA integrity. Further investigations are required to verify this hypothesis.

In summary, our data provides evidence that biallelic mutations in $Y R D C$ result in a severe developmental disorder with progeroid features and might be linked to reduced 
A

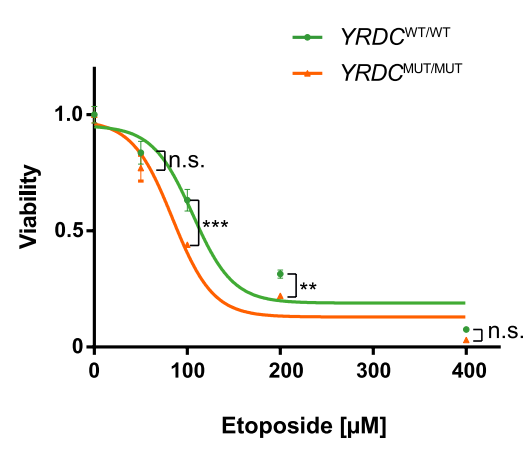

B

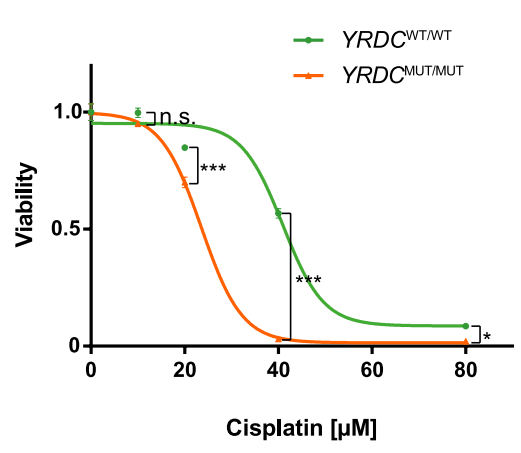

C

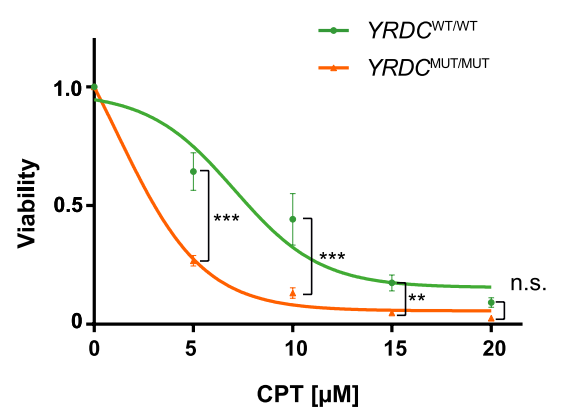

D

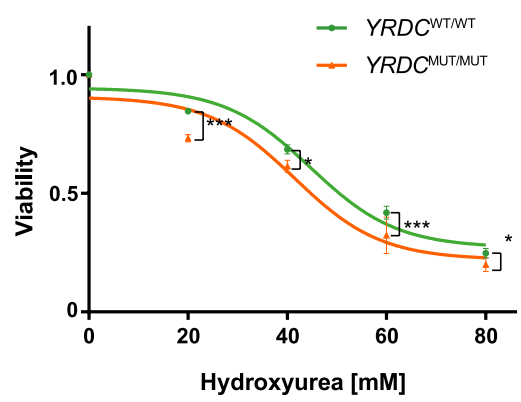

E

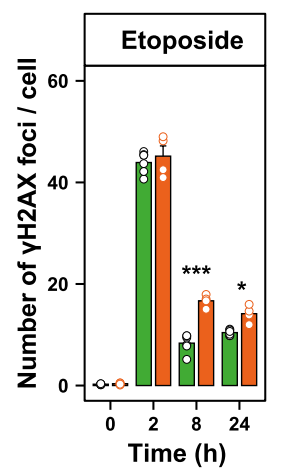

$\mathbf{F}$

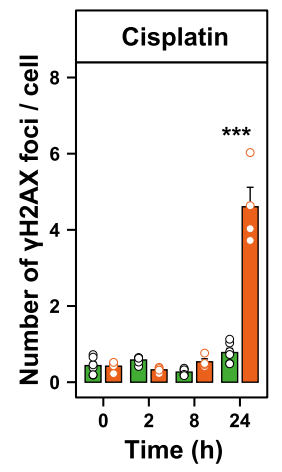

G

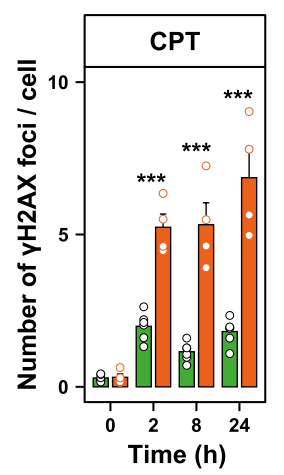

H

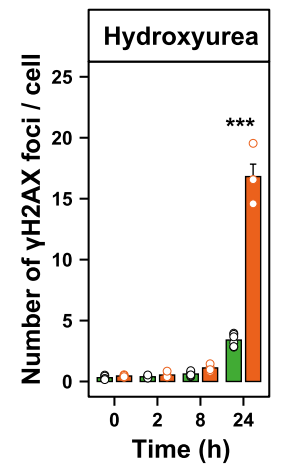

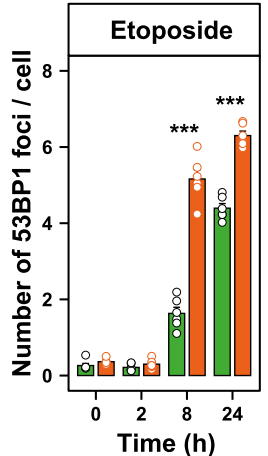

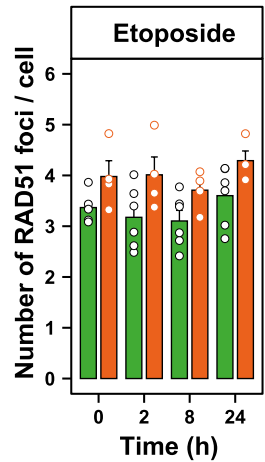

OYRDCWTMT - YRDC ${ }^{\text {MUTMUT }}$
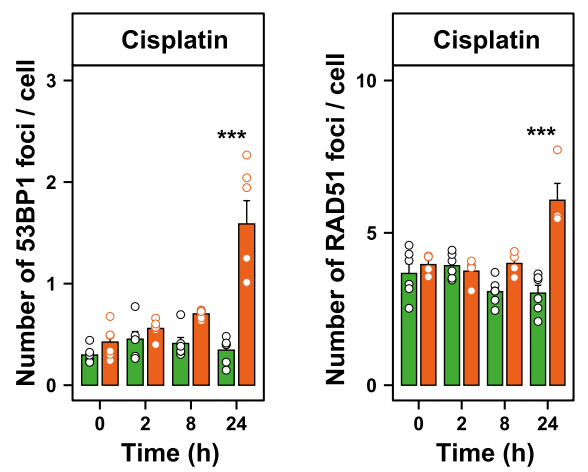

OYRDCWTWT - YRDCMUT/MUT
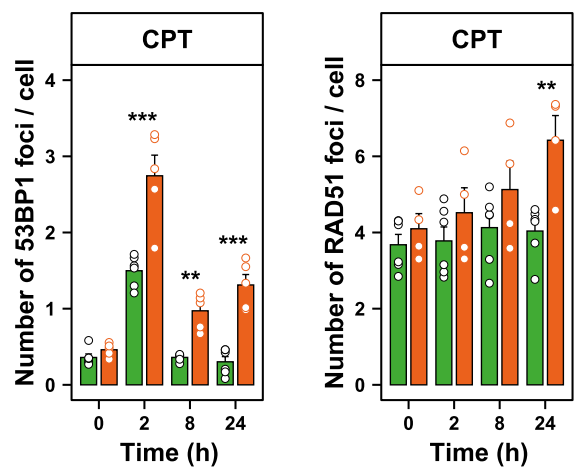

- YRDC ${ }^{W T} / \mathrm{WT}$

- YYRDCMUTMUT

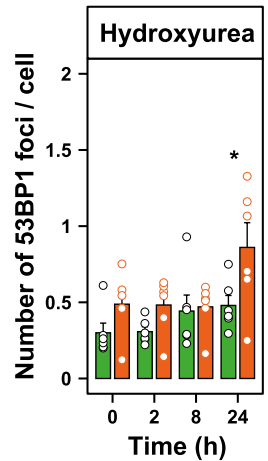

OYRDCWTWT

- YYRDC ${ }^{\text {MUT/MUT }}$ 
4Fig. 4 Homozygous c.662T $>\mathrm{C}$ mutation in $Y R D C$ confers cellular sensitivity to genotoxic agents and a DSB repair defect. A-D Cells of the indicated genotypes were treated with etoposide (A), cisplatin (B), CPT (C) or HU (D) for $96 \mathrm{~h}$ and viability was assessed by CellTiterGlo (CTG) assays. Error bars represent SD of the mean of three technical replicates with $>2000$ cells/well. $p$ values were calculated using $t$ test with Welch's correction not assuming equal variance. ${ }^{*} p<0.05, * * p<0.01, * * * p<0.001$. E-H Cells of the indicated genotype were treated for $2 \mathrm{~h}$ with etoposide $(150 \mu \mathrm{M})(\mathbf{E})$, cisplatin $(20 \mu \mathrm{M})(\mathbf{F})$, CPT $(2 \mu \mathrm{M})(\mathbf{G})$ or with HU $(20 \mathrm{mM})(\mathbf{H})$ and stained for $\gamma \mathrm{H} 2 \mathrm{AX}$ (left panel), 53BP1 (middle panel) and RAD51 (right panel). Total number of $\gamma \mathrm{H} 2 \mathrm{AX}, 53 \mathrm{BP} 1$ and RAD51 foci per cell were quantified. Dots indicate means of separate replicates; $n=3$ wells; $>2000$ cells/well; experiments were independently performed three times; two-way ANOVA with Bonferroni's test. ${ }^{*} p<0.05$, $* * p<0.01, * * * p<0.001$ levels of t6A modifications as well as telomere shortening and alteration of DNA damage response.

\section{Methods}

\section{Whole-exome sequencing}

Whole-exome sequencing on DNA of the patient II.5 and both parents was performed using the Agilent SureSelectXT Human All Exon V7 enrichment kit on an Illumina HiSeq 4000 sequencer. The "Varbank 2" pipeline of the Cologne Center for Genomics (CCG) was used to analyze the exome data using the following filter criteria: coverage of $>6$ reads, quality score of $>10$, allele frequency $\geq 25 \%$, and a minor allele frequency $(\mathrm{MAF})<0.1 \%$ in the gnomAD (https://

Table 1 Summary of the clinical findings in our patient and the affected individuals with biallelic variants in YRDC reported by Arrondel et al. (2019)

\begin{tabular}{|c|c|c|c|c|}
\hline \multirow{3}{*}{$\begin{array}{l}\text { Family } \\
\text { ID }\end{array}$} & \multirow{3}{*}{$\begin{array}{l}\text { Reported here } \\
\text { A } \\
\text { Patient } 1\end{array}$} & \multicolumn{3}{|l|}{ Arrondel et al. (2019) } \\
\hline & & \multirow{2}{*}{$\begin{array}{l}\text { B } \\
\text { Patient } 2\end{array}$} & \multicolumn{2}{|l|}{$\mathrm{C}$} \\
\hline & & & Patient 3 & Patient 4 \\
\hline Variant & c.662T > C; (p.Ile221Thr) & $\begin{array}{l}\text { c. } 251 \mathrm{C}>\text { T/c.721_724del; } \\
\text { (p.Ala84Val)/ } \\
\text { (p.Val2411lefs*72) }\end{array}$ & $\begin{array}{l}\text { c.794_796del; } \\
\text { (p.Leu265del) }\end{array}$ & $\begin{array}{l}\text { c.794_796del; } \\
\text { (p.Leu265del) }\end{array}$ \\
\hline Zygosity & Homozygous & Compound heterozygous & Homozygous & Homozygous \\
\hline Exon & 4 & $1 / 4$ & 4 & 4 \\
\hline Gender & Male & Female & Male & Female \\
\hline Ancestry & Northern Iraq & European & European & \\
\hline Consanguinity & Distantly & No & Yes & \\
\hline \multicolumn{5}{|l|}{ Clinical manifestations } \\
\hline Birth & Born at $37+0$ weeks & ND & ND & ND \\
\hline Birth length & $46 \mathrm{~cm}(-1.8 \mathrm{SD})$ & ND & ND & ND \\
\hline Birth weight & $1740 \mathrm{~g}(-3.1 \mathrm{SD})$ & ND & ND & ND \\
\hline $\begin{array}{l}\text { Head circumference at } \\
\text { birth }\end{array}$ & $29 \mathrm{~cm}(-3.6 \mathrm{SD})$ & ND & ND & ND \\
\hline Seizures & + & - & - & - \\
\hline Kidney dysfunction & + & + & + & + \\
\hline Liver dysfunction & + & - & - & - \\
\hline Hypothyroidism & + & + & + & + \\
\hline Facial dysmorphisms & $\begin{array}{l}\text { Progeroid appearance, } \\
\text { low-set and large appear- } \\
\text { ing ears, micro- and } \\
\text { retrognathia, long, smooth } \\
\text { philtrum, and wrinkled } \\
\text { skin }\end{array}$ & + & + & + \\
\hline Brain anomaly & Primary microcephaly & Secondary microcephaly & Primary microcephaly & Primary microcephaly \\
\hline Skeletal & $\begin{array}{l}\text { Arachnodactyly, adducted } \\
\text { thumbs }\end{array}$ & - & Arachnodactyly & Arachnodactyly \\
\hline Intestinal malrotation & + & - & - & - \\
\hline Age at death & 12 days & 15 months & 1.5 months & 3 months \\
\hline
\end{tabular}

+ Present, - Absent, ND Not documented, SD Standard deviation 
gnomad.broadinstitute.org) database. The following databases were used to obtain gene information: National Center for Biotechnology Information (NCBI; https://www.ncbi. nlm.nih.gov), Ensembl Genome Server (http://www.ensem bl.org), UCSC Genome Bioinformatics (http://genome-euro. ucsc.edu) and Genome Aggregation Database (gnomAD; http://gnomad.broadinstitute.org). The variant identified by whole-exom sequencing was amplified from DNA of the index patient and PCR products were sequenced by BigDye Terminator method on an ABI 3500XL sequencer (Life Technologies, Germany). The identified mutation was resequenced in an independent experiment and tested for cosegregation within the family.

\section{Cell lines and cell cultures}

Primary dermal fibroblasts established from patient II.5 and respective controls were cultured in Dulbecco's modified Eagle medium (DMEM, Gibco) supplemented with $10 \%$ fetal calf serum (FCS, Gibco), and antibiotics. All functional assays presented in the manuscript were performed using cell lines that were matched for passage number and, where possible, age as well as sex.

\section{Protein isolation and analysis}

Primary dermal fibroblasts were solubilized using ice-cold RIPA buffer (10 mM Tris, pH: 8.0; $150 \mathrm{mM} \mathrm{NaCl} ; 1 \mathrm{mM}$ EDTA; 1\% NP-40; protease inhibitors P 2714 [SigmaAldrich, USA]). The total protein concentration of extracts was determined using the BCA Protein Assay Kit (Thermo Fisher Scientific, USA). 15-25 $\mu$ g of total cell lysates were separated by Mini-PROTEAN ${ }^{\circledR}$ TGX $^{\text {TM }}$ Precast Gels (BioRad, Germany) and blotted onto nitrocellulose membranes (Bio-Rad, Germany). Protein detection was performed using an antibody to YRDC (Santa Cruz Biotechnology Inc., USA). Secondary antibodies conjugated to peroxidase (Santa Cruz Biotechnology Inc., USA) were used and blots were developed using an enhanced chemiluminescence system, Clarity Max Western (Bio-Rad, Germany), followed by detection using the ChemiDoc ${ }^{\mathrm{TM}}$ Touch Imaging System (Bio-Rad, Germany).

\section{Immunofluorescence stainings and fluorescence measurements}

For immunofluorescence detection of YRDC, primary dermal fibroblasts were grown on coverslips, washed with PBS, fixed with $4 \%$ paraformaldehyde for $15 \mathrm{~min}$ and permeabilized with $1 \%$ Triton X-100 for $10 \mathrm{~min}$. Upon blocking for 30 min with $3 \%$ BSA in PBS, slides were incubated with YRDC antibodies (1:500, Santa Cruz Biotechnology Inc) overnight. Slides were washed with PBS and incubated with
Alexa Fluor ${ }^{\circledR} 488$ conjugated goat-anti-rabbit antibodies (Thermo Fisher Scientific) for $1 \mathrm{~h}$. Subsequently, slides were washed with PBS, mounted in Vectashield with DAPI (H 1200; Vector Laboratories) and viewed with an Olympus FLUOVIEW FV 1000 confocal laser scanning microscope.

For automated immunofluorescence microscopy, human dermal fibroblasts were seeded onto 96-well plates. For RAD51 stainings, cells were additionally pre-extracted in sucrose buffer (25 mM HEPES pH 7.5, $50 \mathrm{mM} \mathrm{NaCl}, 1 \mathrm{mM}$ EDTA, $3 \mathrm{mM} \mathrm{MgCl} 2,300 \mathrm{mM}$ sucrose, $0.5 \%$ Triton-X) for $2 \mathrm{~min}$ on ice. Cells were fixed for $10 \mathrm{~min}$ in room temperature with $4 \%$ paraformaldehyde, followed by three washes with ice-cold $1 \times$ PBS. Subsequently, cells were blocked for 60 min at room temperature in PBS containing 5\% normal goat serum (NGS), $2 \%$ bovine serum albumin and $0.01 \%$ Triton X-100. Incubation with the primary antibody was performed over night at $4{ }^{\circ} \mathrm{C}$. Incubation with the secondary antibody was performed for $1 \mathrm{~h}$ at room temperature. Analysis was performed as described below in "High-content screening microscopy."

\section{Quantification of $\mathrm{t}^{6} \mathrm{~A}$ modification}

$500 \mathrm{ng}$ of total RNA per sample was digested to nucleosides using $0.6 \mathrm{U}$ nuclease P1 from P. citrinum (Sigma-Aldrich), $0.2 \mathrm{U}$ snake venom phosphodiesterase from $C$. adamanteus (Worthington), 2 U FastAP (Thermo Scientific), $10 \mathrm{U}$ benzonase (Sigma-Aldrich), 200 ng Pentostatin (Sigma-Aldrich) and $500 \mathrm{ng}$ Tetrahydrouridine (Merck-Millipore) in $25 \mathrm{mM}$ ammonium acetate ( $\mathrm{pH} 7.5$; Sigma-Aldrich) over night at $37^{\circ} \mathrm{C}$. The nucleosides were then spiked with internal standard $\left({ }^{13} \mathrm{C}\right.$ stable isotope-labeled nucleosides from $S$. cerevisiae) and subjected to analysis. $100 \mathrm{ng}$ of digested RNA and $50 \mathrm{ng}$ internal standard were analyzed via LC-MS [Agilent 1260 series and Agilent 6460 Triple Quadrupole mass spectrometer equipped with an electrospray ion source (ESI)]. The solvents consisted of $5 \mathrm{mM}$ ammonium acetate buffer (pH 5.3; solvent A) and LC-MS grade acetonitrile (solvent B; Honeywell). The elution started with $100 \%$ solvent A with a flow rate of $0.35 \mathrm{ml} / \mathrm{min}$, followed by a linear gradient to $8 \%$ solvent B at $10 \mathrm{~min}$ and $40 \%$ solvent B after $20 \mathrm{~min}$. Initial conditions were regenerated with $100 \%$ solvent A for $10 \mathrm{~min}$. The column used was a Synergi Fusion $(4 \mu \mathrm{M}$ particle size, $80 \AA$ pore size, $250 \times 2.0 \mathrm{~mm}$; Phenomenex). The UV signal at $254 \mathrm{~nm}$ was recorded via a diode array detector (DAD) to monitor the main nucleosides. ESI parameters were as follows: gas temperature $350^{\circ} \mathrm{C}$, gas flow $81 / \mathrm{min}$, nebulizer pressure $50 \mathrm{psi}$, sheath gas temperature $350{ }^{\circ} \mathrm{C}$, sheath gas flow $12 \mathrm{l} / \mathrm{min}$, capillary voltage $3000 \mathrm{~V}$. The MS was operated in the positive ion mode using Agilent MassHunter software in the dynamic MRM (multiple reaction monitoring) mode. For relative quantification, an internal 
calibration was applied as described previously (Kellner et al. 2014).

\section{Single-cell RNA sequencing (scRNA-seq)}

A full-length, single-cell RNA-seq approach was used with the ICELL8 Single-Cell System. Cell suspensions were fluorescently labeled with live/dead stain (DAPI and Texas Red) for 15 min prior to their dispensing into the 5184 microchip nanowells (ICELL8 System). After selection of wells containing single, live cells, cDNA was synthesized via oligo-dT priming in a one-step RT-PCR reaction. P5 indexing primers for subsequent library preparation were indexed into all wells, with each of the 72 rows on the ICELL8 chip receiving a different index, in addition to Terra polymerase and reaction buffer. Transposition enzyme and reaction buffer ( $\operatorname{Tn} 5$ mixture) were dispensed to selected wells, and the transposition reaction was performed. P7 indexing primers were dispensed to wells, with each of the 72 columns on the chip receiving a different index. Final Illumina libraries were amplified and pooled as they are extracted from the chip. Pooled libraries were purified and size selected using Agencourt AMPure XP magnetic beads (Beckman Coulter) to obtain an average library size of $500 \mathrm{bp}$. A typical yield for a library comprised of $\sim 1300$ cells was $\sim 15 \mathrm{nM}$. Libraries were sequenced on the HiSeq 4000 (Illumina) to obtain on average $\sim 0.5$ Mio reads per cell (SE; $50 \mathrm{bp}$ ).

\section{Data pre-processing and quality control}

Raw sequencing files (bcl) were demultiplexed and fastq files were generated using Illumina bcl2fastq software (v2.20.0). For data pre-processing, the ICELL8 mappa analysis pipeline (version 0.9) was run. In brief, mappa_demuxer was used to allocate the reads to the cells based on the cell barcodes provided in the well-list file. Subsequently, the mappa_analyser was used as a wrapper function to perform read trimming with cutadapt (version 2.5), genome alignment to Homo sapiens genome GRCh38 using STAR (version 2.7.2b), read counting for exonic, genomic and mitochondrial regions using featureCounts (version 1.6.4) and summarization using the $\mathrm{R}$ package hanta (version 1.0.0). As part of the hanta package, the final matrix of reads counts for all genes in all cells underwent quality control (QC) filtering for low expressions using default parameters: (a) for cells, only those with at least 10,000 reads associated to at least 300 different genes were kept, and (b) for genes, only those containing at least 100 reads mapped to them from at least 3 different cells were kept. After applying these quality control filtering criteria, we analyzed 201 out of 256 single patient cells and 428 out of 614 single cells of the two sex- and age-matched controls. The de-regulated genes (abs $(\log 2 \mathrm{FolgChange})>0.25)$ are listed in the supplement (Supplemental Table S2).

\section{Data analysis}

For differential expression (DE) analysis between pairs of samples, the QC-filtered read count matrix was used as input to determine which genes were differentially expressed using Wilcoxon Rank Sum and Signed Rank Tests. For the individual DE results, a rank score was calculated for each gene using the formula rank $=-\log 10(p$-value $) \times \log 2 \mathrm{FC}$, where $p$ value is the raw $p$ value and $\log 2 \mathrm{FC}$ is the $\log 2$ fold-change. The ranks were used as input for an enrichment analysis using WebGestaltR (version 0.4.4) for all gene ontologies and Reactome pathways.

\section{Cell viability measurement}

Cell lines were plated into 384-well plates at densities of 5000 cells/well. $24 \mathrm{~h}$ later, cells were treated with various doses of genotoxic chemicals for $96 \mathrm{~h}$. After incubation, room-temperature CellTiter-Glo® Reagent (Promega, USA) was added 1:1 to each well and the plates were incubated at room temperature for $10 \mathrm{~min}$. Luminescence was measured with the Tecan Infinite M1000 Pro (Tecan, Männedorf, $\mathrm{CHE}$ ) and normalized against control cells treated with vehicle solution. Error bars represent SD of the mean of three independent experiments. $p$ values were calculated using $t$ test with Welch's correction not assuming equal variance. $* p<0.05, * * p<0.01, * * * p<0.001$.

\section{High-content screening microscopy}

High-content screening (HCS) microscopy was performed on human dermal fibroblasts using a Thermo Fisher Cellomics ArrayScan XTI with LED light source. Images of $1104 \times 1104$ pixels were acquired with a $20 \times$ objective (Zeiss) and analyzed using the Cellomics software package (Colocalization V.4 Bioapplication). Images were background corrected (3D surface fitting) and DAPI stained cell nuclei were identified according to the object selection parameters size: $100-1500 \mu \mathrm{m}^{2}$, ratio of perimeter squared to $4 \pi$ area: $1-2$, length-to-width ratio: $1-5$, average intensity: $400-4000$, total intensity: $4 \times 10^{6}-2 \times 10^{7}$. Foci of $\gamma \mathrm{H} 2 \mathrm{AX}$, 53BP1 and RAD51 were quantified within the nuclear region at another excitation wavelength $(485 \pm 20 \mathrm{~nm}$ or $650 \pm 20 \mathrm{~nm}$ ). Object selection parameters for foci were size: $0.1-10 \mu \mathrm{m}^{2}$, ratio of perimeter squared to $4 \pi$ area: $1-5$, length-to-width ratio: $1-5$, average intensity: $500-10,000$, total intensity: $300-2 \times 10^{6}$. HCS microscopy results are means \pm standard deviation; $>2000$ cells/well; three technical replicates were measured per experiment; two-way 
ANOVA with Bonferroni's test. $* p<0.05, * * p<0.01$, $* * * p<0.001$.

\section{Q-FISH-based evaluation of telomere length from primary dermal fibroblasts}

For preparation of cells for Q-FISH analysis, primary cell cultures of human dermal fibroblasts were maintained in 75TC flasks up to passage 5, then split and harvested $48 \mathrm{~h}$ later at approximately $75 \%$ confluency. Therefore, cells were trypsinized for 1-2 min, trypsin was neutralized by adding media, and cells were pelleted ( $5 \mathrm{~min}, 200 \mathrm{~g}$ ). Supernatants were completely removed and $10 \mathrm{ml}$ of pre-warmed $\left(37^{\circ} \mathrm{C}\right)$ hypotonic solution $(0.0025 \mathrm{M} \mathrm{Na}$ citrate, $0.04 \mathrm{M} \mathrm{KCl})$ was dropwise added to pellets while vortexing slowly. Cells were incubated for $15 \mathrm{~min}$ at $37{ }^{\circ} \mathrm{C}$ and centrifuged $(5 \mathrm{~min}, 200 \mathrm{~g}$, room temperature). Supernatants were partly removed leaving approximately $1 \mathrm{ml}$ in the tubes.

For fixation, cell pellets slowly resuspended by vortexing. $10 \mathrm{ml}$ of cold Carnoy fixative (75\% methanol, $25 \%$ acetic acid) was added dropwise to the pellets while vortexing with increasing speed. Then cells were pelleted at $(5 \mathrm{~min}$, $200 \mathrm{~g}$, room temperature) and supernatants removed leaving approximately $1 \mathrm{ml}$ in the tubes. The fixation/centrifugation steps were repeated three times. Cells were resuspended in a small volume of fixative and cell suspension dropped onto clean superfrost slides at optimal ambient humidity. Slides were air-dried and spreading efficiencies were checked by light microscopy. Slides with cell spreads were dried overnight.

Pretreatment slides were rehydrated for $15 \mathrm{~min}$ in PBS, fixed in $4 \%$ formaldehyde in PBS for 4 min at $37{ }^{\circ} \mathrm{C}$, washed twice in PBS for 5 min at $37{ }^{\circ} \mathrm{C}$ and air-dried. $100 \mu \mathrm{l}$ of RNase A solution $(100 \mu \mathrm{g} / \mathrm{ml}$ in $2 \times \mathrm{SSC})$ per slide was added and slides were incubated for $1 \mathrm{~h}$ at $37{ }^{\circ} \mathrm{C}$ in a Thermobrite incubator (a programmable temperature controlled slide processing system for FISH procedures). Afterwards the slides were washed three times in $2 \times$ SSC and once in distilled water. The slides were immersed in $0.005 \%$ Pepsin for $4 \mathrm{~min}$ at $37{ }^{\circ} \mathrm{C}$ for protein digestion, washed twice in PBS for $3 \mathrm{~min}$ at $37{ }^{\circ} \mathrm{C}$ and fixed in $4 \%$ formaldehyde in PBS for 4 min at $37^{\circ} \mathrm{C}$. The slides were washed twice in PBS for $5 \mathrm{~min}$ at $37^{\circ} \mathrm{C}(\times 2)$ and once in PBS for $5 \mathrm{~min}$ at room temperature. Afterwards the slides were sequentially dehydrated for $2 \min$ in $70 \%, 85 \%, 100 \%$ ethanol, respectively, and air-dried.

\section{Denaturation and PNA hybridization}

For denaturation and PNA hybridization, slides were placed in a pre-heated Thermobrite at $80{ }^{\circ} \mathrm{C}$ for $5 \mathrm{~min} .100 \mu \mathrm{l}$ of pre-heated PNA probe (TelC Star635P, $200 \mathrm{nM}$, Eurogentec) in hybridization solution (Oxford Gene Technology) was distributed per slide, overlaid with a coverslip and denatured at $80{ }^{\circ} \mathrm{C}$ for $5 \mathrm{~min}$ in the dark. The slides were placed in a humidified hybridization chamber at $4{ }^{\circ} \mathrm{C}$ overnight for PNA probe hybridization (the coverslips were fixed with silicon glue).

Afterwards, slides were immersed in washing solution $(2 \times$ SSC, $1 \%$ Tween-20) at room temperature to remove the coverslip and silicon glue and washed twice in washing solution for $10 \mathrm{~min}$ at $55-60^{\circ} \mathrm{C}$, afterwards washed twice in $2 \times$ SSC and once in distilled water. After drying on air in the dark a drop of mounting media (Prolong Gold Antifade Reagent with DAPI, Invitrogen) was put onto slide, the slide was carefully covered with a coverslip and incubated in the dark overnight.

\section{Q-FISH microscopy}

FISH slides were imaged in confocal and STED mode using a custom-built setup based on an Olympus IX83 inverted microscope with an Abberior QUAD scanner. Slides were mounted on a $100 \times 1.4$ NA oil-immersion objective and intact nuclei were identified in the DAPI channel. DAPI and TelC-Star635P signals were excited at $405 \mathrm{~nm}$ and $633 \mathrm{~nm}$ excitation wavelengths and recorded with $422-467 \mathrm{~nm}$ and 650-720 nm emission filters, respectively. 3D-Confocal imaging was performed at $80 \mathrm{~nm} \times 80 \mathrm{~nm}-200 \mathrm{~nm}$ pixel size $(x, y, z)$ at $100 \mu$ s pixel dwell time. In addition, single frames of imaging planes including bright TelC-Star635P signal spots were acquired using STED mode to confirm spatial separation of telomeres in confocal imaging.

\section{Q-FISH image analysis}

In ImageJ Fiji, Images were manually masked by the DAPI signal to exclude background signals outside nuclei. TelCStar635P signals were smoothed by a Gaussian filter with $\sigma=80 \mathrm{~nm}$ and subjected to a 3D spot detection routine (Herbert et al. 2014) with a background threshold of 25 counts and a minimum spot size of 10 voxels. The retrieved center of mass coordinates for each telomere spot were used to center two orthogonal lateral fits of the signal by a Gaussian function. The better-fit result was used to retrieve telomere brightness from its amplitude, when at least one fit was sufficient $\left(r^{2}>0.8\right)$. No further image or data filtering was applied.

Supplementary Information The online version contains supplementary material available at https://doi.org/10.1007/s00439-021-02347-3.

Acknowledgements We are grateful to all family members that participated in this study and Karin Boss for critically reading the manuscript.

Author contributions JS and BW designed the study. JS, RJ, TK, SEL, GY and BW designed the experiments. JS, JG, TP, AK, NS, MS, GW, 
JA, HT, PN, JI, YL, CM, BL, HCR, TH, GS, MH, RJ, DW, TK, SEL and GY performed the experiments and analyzed the data. JS, GY and BW wrote and edited the manuscript. TP, AK, MS, GS, RJ, TK and SEL contributed to the manuscript.

Funding Open Access funding enabled and organized by Projekt DEAL. This work was supported by the European Reference Network ITHACA; the Deutsche Forschungsgemeinschaft (DFG, German Research Foundation) under Research Group FOR 2800 "Chromosome Instability: Cross-talk of DNA replication stress and mitotic dysfunction" and Germany's Excellence Strategy, Cluster of Excellence "Multiscale Bioimaging: from Molecular Machines to Networks of Excitable Cells" (MBExC; EXC 2067/1-390729940) to B. Wollnik and S.E. Lehnart; the SFB 1002 projects A09 and S02 to S.E. Lehnart; and the SPP1784 to M. Helm. A customized upgrade of the STED imaging setup for telomere nanoscopy setup was funded by DZHK (German Centre for Cardiovascular Research) to S.E. Lehnart. S.E. Lehnart was supported by the Transatlantic Network of Excellence CURE-PLaN, Fondation Leducq Paris, France.

Data availability statement The data that support the findings of this study are available on request from the corresponding author. The data are not publicly available due to privacy or ethical restrictions.

Code availability Not applicable.

\section{Declarations}

Conflict of interest The authors have declared that no conflict of interest exists.

Ethical approval The study was approved by the Ethics Committee of University Medical Center Göttingen (approval number 3/2/16) and performed in accordance with the Declaration of Helsinki protocols.

Informed consent to participate Written informed consent was obtained from all participants or their legal representatives prior to the participation in the study, and DNA from participating family members was extracted from peripheral blood lymphocytes by standard extraction procedures.

Informed consent for publication Consent for publication was obtained from all participants.

Open Access This article is licensed under a Creative Commons Attribution 4.0 International License, which permits use, sharing, adaptation, distribution and reproduction in any medium or format, as long as you give appropriate credit to the original author(s) and the source, provide a link to the Creative Commons licence, and indicate if changes were made. The images or other third party material in this article are included in the article's Creative Commons licence, unless indicated otherwise in a credit line to the material. If material is not included in the article's Creative Commons licence and your intended use is not permitted by statutory regulation or exceeds the permitted use, you will need to obtain permission directly from the copyright holder. To view a copy of this licence, visit http://creativecommons.org/licenses/by/4.0/.

\section{References}

Acuna-Hidalgo R, Veltman JA, Hoischen A (2016) New insights into the generation and role of de novo mutations in health and disease. Genome Biol 17(1)
Arrondel C, Missoury S, Snoek R, Patat J, Menara G, Collinet B, Liger D, Durand D, Gribouval O, Boyer O, Buscara L, Martin G, Machuca E, Nevo F, Lescop E, Braun DA, Boschat A-C, Sanquer S, Guerrera IC, Revy P, Parisot M, Masson C, Boddaert N, Charbit M, Decramer S, Novo R, Macher M-A, Ranchin B, Bacchetta J, Laurent A, Collardeau-Frachon S, van Eerde AM, Hildebrandt F, Magen D, Antignac C, van Tilbeurgh H, Mollet G (2019) Defects in t6A tRNA modification due to GON7 and YRDC mutations lead to Galloway-Mowat syndrome. Nat Commun 10(1):3967

Blackburn EH (1991) Structure and function of telomeres. Nature 350(6319):569-573

Braun DA, Rao J, Mollet G, Schapiro D, Daugeron M-C, Tan W, Gribouval O, Boyer O, Revy P, Jobst-Schwan T, Schmidt JM, Lawson JA, Schanze D, Ashraf S, Ullmann JFP, Hoogstraten CA, Boddaert N, Collinet B, Martin G, Liger D, Lovric S, Furlano M, Guerrera IC, Sanchez-Ferras O, Hu JF, Boschat A-C, Sanquer S, Menten B, Vergult S, de Rocker N, Airik M, Hermle T, Shril S, Widmeier E, Gee HY, Choi W-I, Sadowski CE, Pabst WL, Warejko JK, Daga A, Basta T, Matejas V, Scharmann K, Kienast SD, Behnam B, Beeson B, Begtrup A, Bruce M, Ch'ng G-S, Lin S-P, Chang J-H, Chen C-H, Cho MT, Gaffney PM, Gipson PE, Hsu C-H, Kari JA, Ke Y-Y, Kiraly-Borri C, Lai W-M, Lemyre E, Littlejohn RO, Masri A, Moghtaderi M, Nakamura K, Ozaltin F, Praet M, Prasad C, Prytula A, Roeder ER, Rump P, Schnur RE, Shiihara T, Sinha MD, Soliman NA, Soulami K, Sweetser DA, Tsai W-H, Tsai J-D, Topaloglu R, Vester U, Viskochil DH, Vatanavicharn N, Waxler JL, Wierenga KJ, Wolf MTF, Wong S-N, Leidel SA, Truglio G, Dedon PC, Poduri A, Mane S, Lifton RP, Bouchard M, Kannu P, Chitayat D, Magen D, Callewaert B, van Tilbeurgh H, Zenker M, Antignac C, Hildebrandt F (2017) Mutations in KEOPS-complex genes cause nephrotic syndrome with primary microcephaly. Nat Genet 49(10):1529-1538

Carrero D, Soria-Valles C, López-Otín C (2016) Hallmarks of progeroid syndromes: lessons from mice and reprogrammed cells. Dis Model Mech 9(7):719-735

Dasari S, Tchounwou PB (2014) Cisplatin in cancer therapy: molecular mechanisms of action. Eur J Pharmacol 740:364-378

Daugeron M-C, Lenstra TL, Frizzarin M, El Yacoubi B, Liu X, BaudinBaillieu A, Lijnzaad P, Decourty L, Saveanu C, Jacquier A, Holstege FCP, de Crécy-Lagard V, van Tilbeurgh H, Libri D (2011) Gcn 4 misregulation reveals a direct role for the evolutionary conserved EKC/KEOPS in the t6A modification of tRNAs. Nucleic Acids Res 39(14):6148-6160

de Lange T (2005) Shelterin: the protein complex that shapes and safeguards human telomeres. Genes Dev 19(18):2100-2110

Di Micco R, Krizhanovsky V, Baker D, Di d'Adda FF (2021) Cellular senescence in ageing: from mechanisms to therapeutic opportunities. Nat Rev Mol Cell Biol 22(2):75-95

Downey M, Houlsworth R, Maringele L, Rollie A, Brehme M, Galicia S, Guillard S, Partington M, Zubko MK, Krogan NJ, Emili A, Greenblatt JF, Harrington L, Lydall D, Durocher D (2006) A genome-wide screen identifies the evolutionarily conserved KEOPS complex as a telomere regulator. Cell 124(6):1155-1168

El Yacoubi B, Lyons B, Cruz Y, Reddy R, Nordin B, Agnelli F, Williamson JR, Schimmel P, Swairjo MA, de Crécy-Lagard V (2009) The universal YrdC/Sua5 family is required for the formation of threonylcarbamoyladenosine in tRNA. Nucleic Acids Res 37(9):2894-2909

El Yacoubi B, Hatin I, Deutsch C, Kahveci T, Rousset J-P, Iwata-Reuyl D, Murzin AG, de Crécy-Lagard V (2011) A role for the universal Kae1/Qri7/YgjD (COG0533) family in tRNA modification. EMBO J 30(5):882-893

Gal H, Majewska J, Krizhanovsky V (2021) The intricate nature of senescence in development and cell plasticity. Semin Cancer Biol. S1044-579X(21)00010-9. https://doi.org/10.1016/j.semcancer. 2021.01.004. [Epub ahead of print]. PMID: 33486077 
Gordon LB, Rothman FG, Lopez-Otin C, Misteli T (2014) Progeria: a paradigm for translational medicine. Cell 156(3):400-407

Harley CB, Futcher AB, Greider CW (1990) Telomeres shorten during ageing of human fibroblasts. Nature 345(6274):458-460

He M-H, Liu J-C, Lu Y-S, Wu Z-J, Liu Y-Y, Wu Z, Peng J, Zhou J-Q (2019) KEOPS complex promotes homologous recombination via DNA resection. Nucleic Acids Res 47(11):5684-5697

Herbert AD, Carr AM, Hoffmann E (2014) FindFoci: a focus detection algorithm with automated parameter training that closely matches human assignments, reduces human inconsistencies and increases speed of analysis. PLoS ONE 9(12):e114749

Huang S, Zhu P, Sun B, Guo J, Zhou H, Shu Y, Li Q (2019) Modulation of YrdC promotes hepatocellular carcinoma progression via MEK/ERK signaling pathway. Biomed Pharmacother 114:108859

Jachimowicz RD, Beleggia F, Isensee J, Velpula BB, Goergens J, Bustos MA, Doll MA, Shenoy A, Checa-Rodriguez C, Wiederstein JL, Baranes-Bachar K, Bartenhagen C, Hertwig F, Teper N, Nishi T, Schmitt A, Distelmaier F, Lüdecke H-J, Albrecht B, Krüger M, Schumacher B, Geiger T, Hoon DSB, Huertas P, Fischer M, Hucho T, Peifer M, Ziv Y, Reinhardt HC, Wieczorek D, Shiloh $Y$ (2019) UBQLN4 represses homologous recombination and is overexpressed in aggressive tumors. Cell 176(3):505-519.e22

Karczewski KJ, Francioli LC, Tiao G, Cummings BB, Alföldi J, Wang Q, Collins RL, Laricchia KM, Ganna A, Birnbaum DP, Gauthier LD, Brand H, Solomonson M, Watts NA, Rhodes D, Singer-Berk M, England EM, Seaby EG, Kosmicki JA, Walters RK, Tashman K, Farjoun Y, Banks E, Poterba T, Wang A, Seed C, Whiffin N, Chong JX, Samocha KE, Pierce-Hoffman E, Zappala Z, O'Donnell-Luria AH, Minikel EV, Weisburd B, Lek M, Ware JS, Vittal C, Armean IM, Bergelson L, Cibulskis K, Connolly KM, Covarrubias M, Donnelly S, Ferriera S, Gabriel S, Gentry J, Gupta N, Jeandet T, Kaplan D, Llanwarne C, Munshi R, Novod S, Petrillo N, Roazen D, Ruano-Rubio V, Saltzman A, Schleicher M, Soto J, Tibbetts K, Tolonen C, Wade G, Talkowski ME; Genome Aggregation Database Consortium, Neale BM, Daly MJ, MacArthur DG (2019) The mutational constraint spectrum quantified from variation in 141,456 humans. Nature 581(7809):434-443. https://doi.org/10.1038/s41586-020-2308-7

Kellner S, Ochel A, Thüring K, Spenkuch F, Neumann J, Sharma S, Entian K-D, Schneider D, Helm M (2014) Absolute and relative quantification of RNA modifications via biosynthetic isotopomers. Nucleic Acids Res 42(18):e142

Lessel D, Kubisch C (2019) Hereditary syndromes with signs of premature aging. Deutsches Arzteblatt International 116(29-30):489-496

Lin H, Miyauchi K, Harada T, Okita R, Takeshita E, Komaki H, Fujioka K, Yagasaki H, Goto Y-I, Yanaka K, Nakagawa S, Sakaguchi Y, Suzuki T (2018) CO2-sensitive tRNA modification associated with human mitochondrial disease. Nat Commun 9(1):1875

Liu Y-Y, He M-H, Liu J-C, Lu Y-S, Peng J, Zhou J-Q (2018) Yeast KEOPS complex regulates telomere length independently of its t6A modification function. J Genet Genomics 45(5):247-257

Martens UM, Zijlmans JM, Poon SS, Dragowska W, Yui J, Chavez EA, Ward RK, Lansdorp PM (1998) Short telomeres on human chromosome 17p. Nat Genet 18(1):76-80

Martínez P, Blasco MA (2011) Telomeric and extra-telomeric roles for telomerase and the telomere-binding proteins. Nat Rev Cancer 11(3):161-176
Meng F-L, Hu Y, Shen N, Tong X-J, Wang J, Ding J, Zhou J-Q (2009) Sua5p a single-stranded telomeric DNA-binding protein facilitates telomere replication. EMBO J 28(10):1466-1478

Meng F-L, Chen X-F, Hu Y, Tang H-B, Dang W, Zhou J-Q (2010) Sua5p is required for telomere recombination in Saccharomyces cerevisiae. Cytometry 20(4):495-498

Muñoz-Espín D, Serrano M (2014) Cellular senescence: from physiology to pathology. Nat Rev Mol Cell Biol 15(7):482-496

Muñoz-Espín D, Cañamero M, Maraver A, Gómez-López G, Contreras J, Murillo-Cuesta S, Rodríguez-Baeza A, Varela-Nieto I, Ruberte J, Collado M, Serrano M (2013) Programmed cell senescence during mammalian embryonic development. Cell 155(5):1104-1118

Olivieri M, Cho T, Álvarez-Quilón A, Li K, Schellenberg MJ, Zimmermann M, Hustedt N, Rossi SE, Adam S, Melo H, Heijink AM, Sastre-Moreno G, Moatti N, Szilard RK, McEwan A, Ling AK, Serrano-Benitez A, Ubhi T, Feng S, Pawling J, Delgado-Sainz I, Ferguson MW, Dennis JW, Brown GW, Cortés-Ledesma F, Williams RS, Martin A, Xu D, Durocher D (2020) A genetic map of the response to DNA damage in human cells. Cell 182(2):481-496

Ourliac-Garnier I, Londoño-Vallejo A (2017) Telomere length analysis by quantitative fluorescent in situ hybridization (Q-FISH). Methods Mol Biol (clifton, N.J.) 1587:29-39

Palomares-Bralo M, Vallespín E, del Pozo Á, Ibañez K, Silla JC, Galán E, Gordo G, Martínez-Glez V, Alba-Valdivia LI, Heath KE, García-Miñaúr S, Lapunzina P, Santos-Simarro F (2017) Pitfalls of trio-based exome sequencing: imprinted genes and parental mosaicism-MAGEL2 as an example. Genet Med 19(11):1285-1286

Perrochia L, Crozat E, Hecker A, Zhang W, Bareille J, Collinet B, van Tilbeurgh H, Forterre P, Basta T (2013) In vitro biosynthesis of a universal t6A tRNA modification in Archaea and Eukarya. Nucleic Acids Res 41(3):1953-1964

Poon SSS, Martens UM, Ward RK, Lansdorp PM (1999) Telomere length measurements using digital fluorescence microscopy. Cytometry 36(4):267-278

Rhinn M, Ritschka B, Keyes WM (2019) Cellular senescence in development, regeneration and disease. Development 146(20):dev151837

Shiloh Y, Lederman HM (2017) Ataxia-telangiectasia (A-T): An emerging dimension of premature ageing. Ageing Res Rev 33:76-88

Srinivasan M, Mehta P, Yu Y, Prugar E, Koonin EV, Karzai AW, Sternglanz R (2011) The highly conserved KEOPS/EKC complex is essential for a universal tRNA modification, t6A. EMBO J 30(5):873-881

Trapp C, Reite K, Klungland A, Epe B (2007) Deficiency of the Cockayne syndrome $\mathrm{B}$ (CSB) gene aggravates the genomic instability caused by endogenous oxidative DNA base damage in mice. EMBO J 26(27):4044-4048

Wu L, Hickson ID (2003) The Bloom's syndrome helicase suppresses crossing over during homologous recombination. Nature 426(6968):870-874

Publisher's Note Springer Nature remains neutral with regard to jurisdictional claims in published maps and institutional affiliations. 


\section{Authors and Affiliations}

\section{Julia Schmidt ${ }^{1}$ (1) - Jonas Goergens ${ }^{2,10} \cdot$ Tatiana Pochechueva $^{3} \cdot$ Annika Kotter $^{4}$ - Niko Schwenzer ${ }^{3,15}$ - Maren Sitte ${ }^{5}$. Gesa Werner ${ }^{1}$. Janine Altmüller ${ }^{6,16,17}$. Holger Thiele ${ }^{6} \cdot$ Peter Nürnberg $^{6}$. Jörg Isensee ${ }^{9} \cdot$ Yun Li $^{1}$. Christian Müller ${ }^{1}$. Barbara Leube $^{7} \cdot$ H. Christian Reinhardt ${ }^{8} \cdot$ Tim Hucho $^{9} \cdot$ Gabriela Salinas $^{5} \cdot$ Mark Helm $^{4} \cdot$ Ron D. Jachimowicz ${ }^{2,10,11,12}$. Dagmar Wieczorek ${ }^{7} \cdot$ Tobias Kohl $^{3,13} \cdot$ Stephan E. Lehnart ${ }^{3,13,14,15,18,19} \cdot$ Gökhan Yigit $^{1}$ • Bernd Wollnik ${ }^{1,15}$}

1 Institute of Human Genetics, University Medical Center Göttingen, Heinrich-Düker-Weg 12, 37073 Göttingen, Germany

2 Max-Planck-Institute for Biology of Ageing, Cologne, Germany

3 Heart Research Center Göttingen, Department of Cardiology and Pneumology, University Medical Center Göttingen, Göttingen, Germany

4 Institute of Pharmaceutical and Biomedical Sciences, Johannes Gutenberg-University Mainz, Mainz, Germany

5 NGS-Integrative Genomics Core Unit (NIG), Institute of Human Genetics, University Medical Center Göttingen, Göttingen, Germany

6 Cologne Center for Genomics (CCG), University of Cologne, Faculty of Medicine and University Hospital Cologne, Cologne, Germany

7 Institute of Human Genetics, Medical Faculty, Heinrich-Heine-University, Düsseldorf, Germany

8 Department of Hematology and Stem Cell Transplantation, University Hospital Essen, University Duisburg-Essen, German Cancer Consortium (DKTK Partner Site Essen), Essen, Germany

9 Department of Anesthesiology and Intensive Care Medicine, Translational Pain Research, University Hospital of Cologne, Cologne, Germany
10 Department I of Internal Medicine, Faculty of Medicine and University Hospital Cologne, Cologne, Germany

11 Cologne Excellence Cluster on Cellular Stress Response in Aging-Associated Diseases, University of Cologne, Cologne, Germany

12 Center for Molecular Medicine Cologne, University of Cologne, Cologne, Germany

13 DZHK (German Centre for Cardiovascular Research), partner site, Göttingen, Germany

14 Collaborative Research Unit SFB 1002, University of Göttingen, Göttingen, Germany

15 Cluster of Excellence "Multiscale Bioimaging: From Molecular Machines to Networks of Excitable Cells" (MBExC), University of Göttingen, Göttingen, Germany

16 Berlin Institute of Health at Charité - Universitätsmedizin Berlin, Core Facility Genomics, Charitéplatz 1, 10117 Berlin, Germany

17 Max Delbrïck Center for Molecular Medicine in the Helmholtz Association (MDC), Berlin, Germany

18 Collaborative Research Unit SFB 1190, University of Göttingen, Göttingen, Germany

19 Transatlantic Network of Excellence CURE-PLaN, Fondation Leducq, Paris, France 